Supporting Information:

\title{
Surface Crystallization of Amorphous Palladium Nanoparticles
}

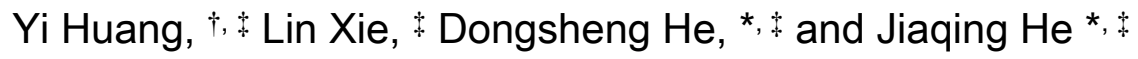 \\ $\dagger$ Department of Physics, Harbin Institute of Technology, Harbin 150001, P. R. China. \\ \$ Department of Physics and Pico Center, Southern University of Science and \\ Technology,
}

Shenzhen 518055, P. R. China.

E-mail (Corresponding authors): heds@sustech.edu.cn; he.jq@sustech.edu.cn 

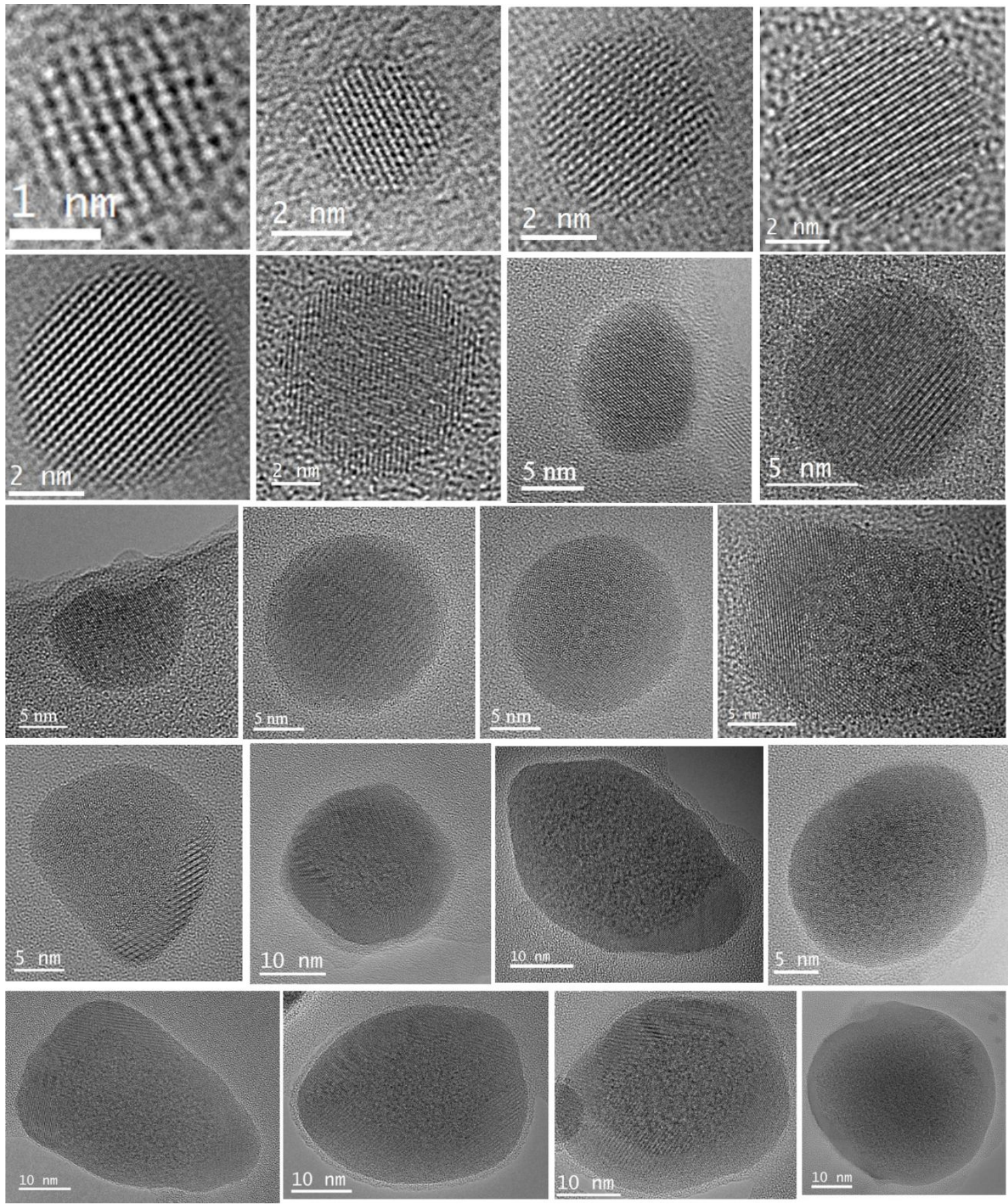

Figure S1. Additional HRTEM images of the nanoparticles experienced strong electron beam irradiation, which was used for analysis and the result is plotted in Figure 2c. The partially crystallization in the surfaces of some nanoparticles can be ascribed to the differences of nucleation sites on the surfaces. 

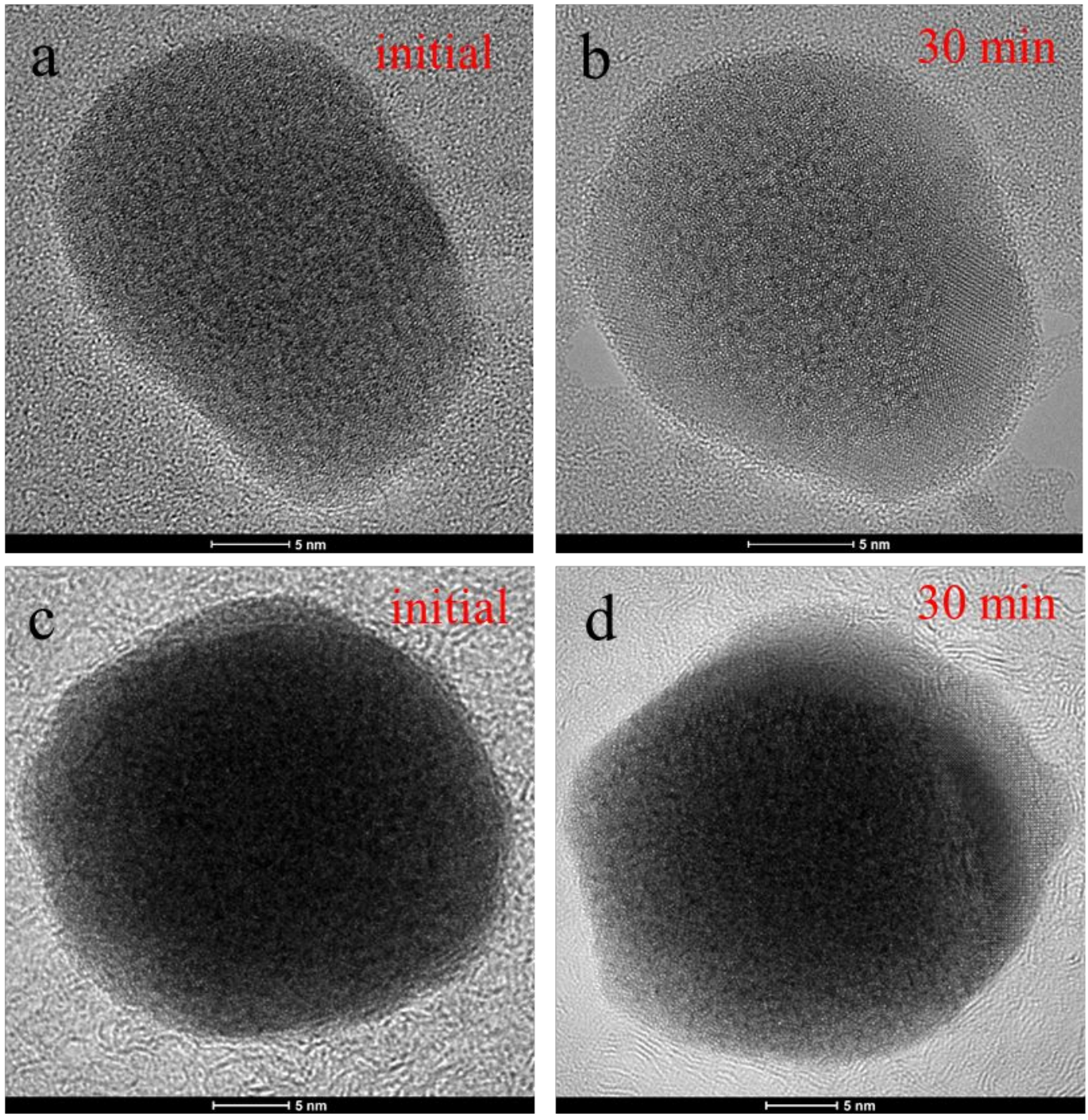

Figure S2. Formation of $L_{e}$ under the incident electron energy of $200 \mathrm{keV}$ (a-b) and $80 \mathrm{keV}$ (c-d) with a dose rate of $11000 \mathrm{e} / \AA^{2} \mathrm{~s}$ and $18600 \mathrm{e} / \AA^{2} \mathrm{~s}$, respectively. The measured average crystalline thicknesses are similar to the thickness induced at $300 \mathrm{kV}$, which are $4.9 \pm 1.5 \mathrm{~nm}$ and $5.6 \pm 1 \mathrm{~nm}$, respectively. 

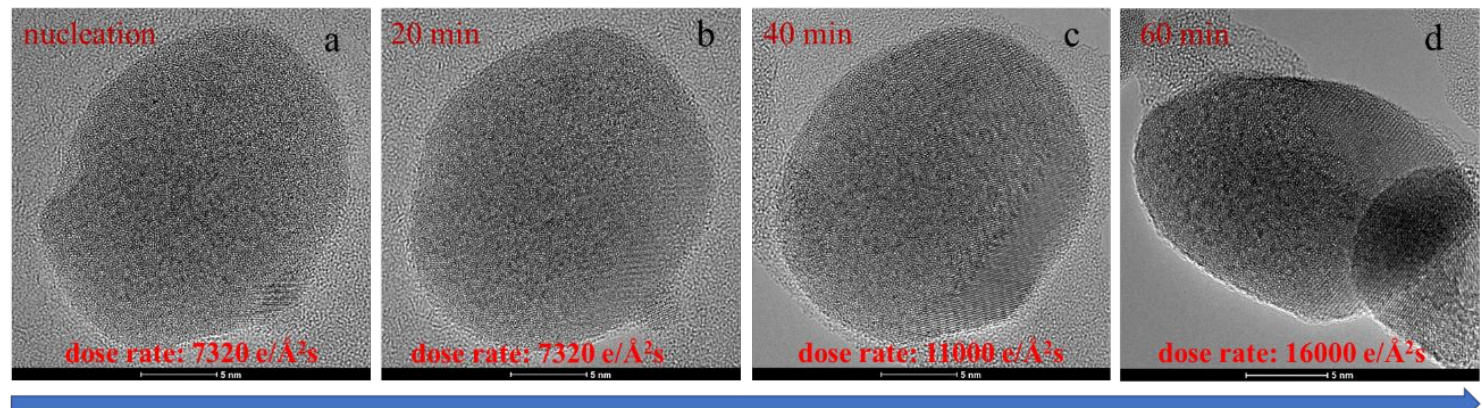

Increasing dose rate

Figure S3. Equilibrium thickness as a function of electron dose rate. With an initial dose rate of $7320 \mathrm{e} / \AA^{2} \mathrm{~s}$, a crystalline layer with an average thickness of $4.5 \pm 0.5 \mathrm{~nm}$ was induced after irradiation of $20 \mathrm{~min}$. Then the same nanoparticle was exposed to a more intensive electron beam for $20 \mathrm{~min}$ at each setup of dose rate $\left(11000 \mathrm{e} / \AA^{2} \mathrm{~s}\right.$ and $16000 \mathrm{e} / \AA^{2} \mathrm{~s}$, respectively). The average crystalline thickness was measured as $5 \pm 0.5 \mathrm{~nm}$ and $4.8 \pm 1 \mathrm{~nm}$, which are consistent with the crystalline thickness triggered by the initial dose rate. These results show that the equilibrium thickness experiences no obvious increase with an increased dose rate from $7320 \mathrm{e} / \AA^{2} \mathrm{~s}$ to 16000 $\mathrm{e} / \AA^{2} \mathrm{~s}$. It should be notable that because the electron beam would induce the rotation of the nanoparticle, significant shape change was observed during prolonged electron beam irradiation (Figure S3d). 


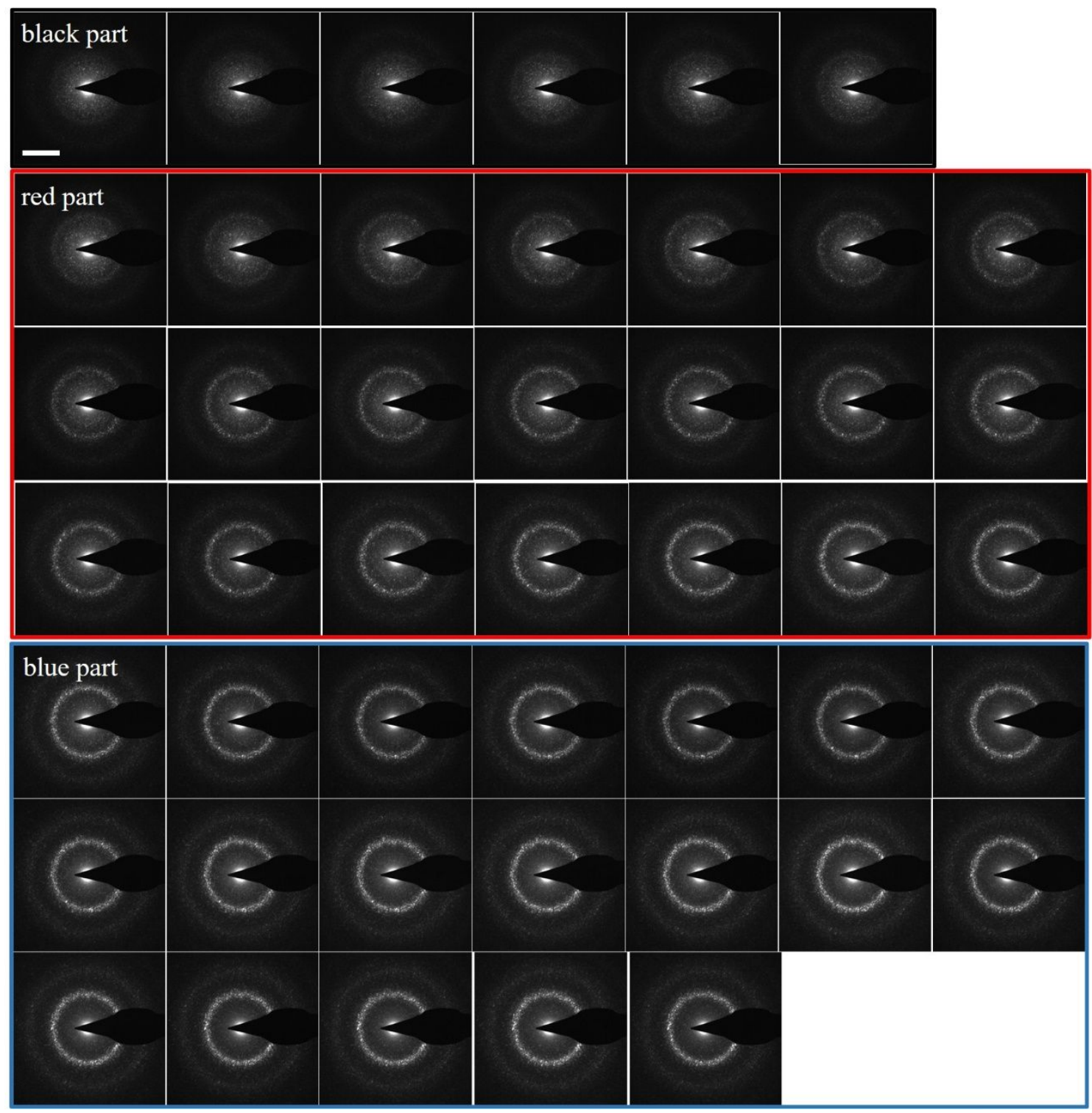

Figure S4. Totally 46 NBD patterns acquiring in a line scan mode. The black, red, and blue parts correspond to the black, red, and blue curves in Figure 4c, respectively. Scale bar: 5 1/nm. 


\section{Schematics of ratio calculation in Figure 4d.}

The value of the ratio was calculated in such a way that is illustrated in Figure S5. Take the ten percent of intensity maximum of the first peak as the baseline, which intersects the original data to form the total width of the peak in this paper. The position of intensity maximum separates the width into two parts. Then,

$$
\text { Ratio }=\frac{\text { the width of the left half peak } \boldsymbol{W}_{\boldsymbol{l}}}{\text { the width of the right half peak } \boldsymbol{W}_{\boldsymbol{r}}}
$$

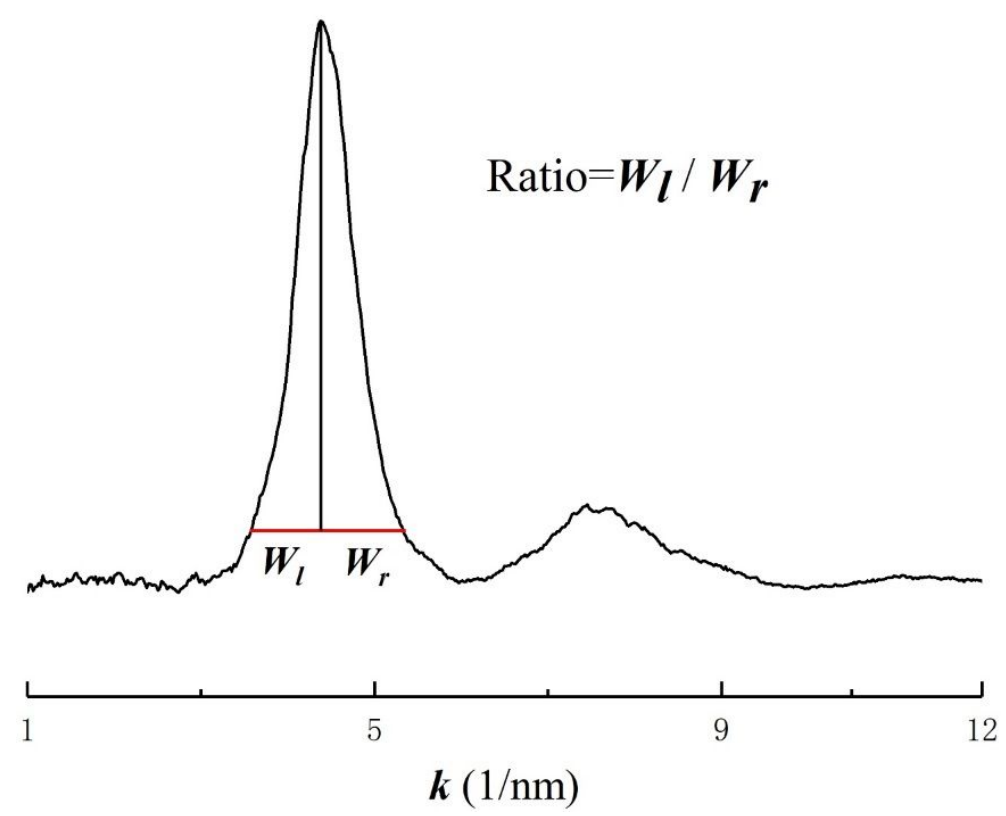

Figure S5. Schematics of ratio calculation in Figure 4d. 


\section{Description of the symmetric decaying model.}

According to the equation written in the main text, the free energy change is described as the following,

$$
\Delta G=\int_{0}^{L_{e}}\left(G_{a c / v}-G_{a / v}\right) d v+4 \pi\left(R-L_{e}\right)^{2} \sigma_{i n t}+4 \pi R^{2} \sigma_{c}-4 \pi R^{2} \sigma_{a}
$$

For the sake of calculation, assuming that

$$
F(l)=\int\left(G_{a c / v}-G_{a / v}\right) d v
$$

here

$$
d v=4 \pi\left(R-L_{e}\right)^{2} d L_{e}
$$

Therefore, by combining equation S2 and S3, the result of the definite integral term in equation S1 is

$$
\int_{0}^{L_{e}}\left(G_{a c / v}-G_{a / v}\right) d v=\int_{0}^{L_{e}}\left(G_{a c / v}-G_{a / v}\right) 4 \pi\left(R-L_{e}\right)^{2} d L_{e}=F\left(L_{e}\right)-F(0)
$$

here

$$
F(0)=0
$$

When the surface crystallization reaches an equilibrium state, $\partial \Delta G / \partial L_{e}=0$, i.e.,

$$
\frac{\partial \Delta G}{\partial L_{e}}=F^{\prime}\left(L_{e}\right)-8 \pi\left(R-L_{e}\right) \sigma_{i n t}=4 \pi\left(R-L_{e}\right)^{2}\left(G_{a c / v}-G_{a / c}\right)-8 \pi\left(R-L_{e}\right) \sigma_{i n t}=0
$$

Equation $\mathrm{S} 6$ describes the relation between $R$ and $L_{e}$ when the equilibrium state has been reached. In this equation, $G_{a c / c}$, which denotes the density of free energy of amorphous-crystalline core-shell structure, is regarded as a constant value within the integration range $\left[0, L_{e}\right]$, i.e. the surface crystallization region. It is notable that two surface energy terms do not contribute to the equation, showing that the surface is not the constraint factor of surface crystallization. As demonstrated in Figure 4 in the main text, the atomic structure of the amorphous Pd nanoparticle gradually varies from the surface to the interior, which allows modeling the density of free energy 
of the amorphous structure $G_{a / v}$ as a gradual decay function from the surface (Figure S6). In the case of a sphere model, it is shown as following,

$$
G_{a / v}=\beta \exp \left(-\mu L_{e}\right)+\beta \exp \left[-\mu\left(2 R-L_{e}\right)\right]+\alpha
$$

where $\beta, \mu$ are the amplitude and rate of decay. The first and the second terms represent the exponentially decaying originated from the near side and far side of the surface, respectively. $\alpha$ denotes the constant background of the function. By substituting equation S7 into equation S6, we get

$4 \pi\left(R-L_{e}\right)^{2}\left\{G_{a c / v}-\beta \exp \left(-\mu L_{e}\right)-\beta \exp \left[-\mu\left(2 R-L_{e}\right)\right]-\alpha\right\}-8 \pi\left(R-L_{e}\right) \sigma_{i n t}=0$

Since the $G_{a c / v}$ and $\alpha$ are both constants, equation $\mathrm{S} 8$ can be further reduced to

$$
4 \pi\left(R-L_{e}\right)^{2}\left\{-\beta \exp \left(-\mu L_{e}\right)-\beta \exp \left[-\mu\left(2 R-L_{e}\right)\right]+\gamma\right\}-8 \pi\left(R-L_{e}\right) \sigma_{\text {int }}=0
$$

where $\gamma$ is the constant difference between $G_{a c / v}$ and $\alpha$. The obtained equation S9 is used to fit the experimental data in Figure $2 \mathrm{c}$, where $\beta, \mu, \gamma$ are free fitting parameters. 
Schematics of the tail overlapping of the symmetric decaying model.

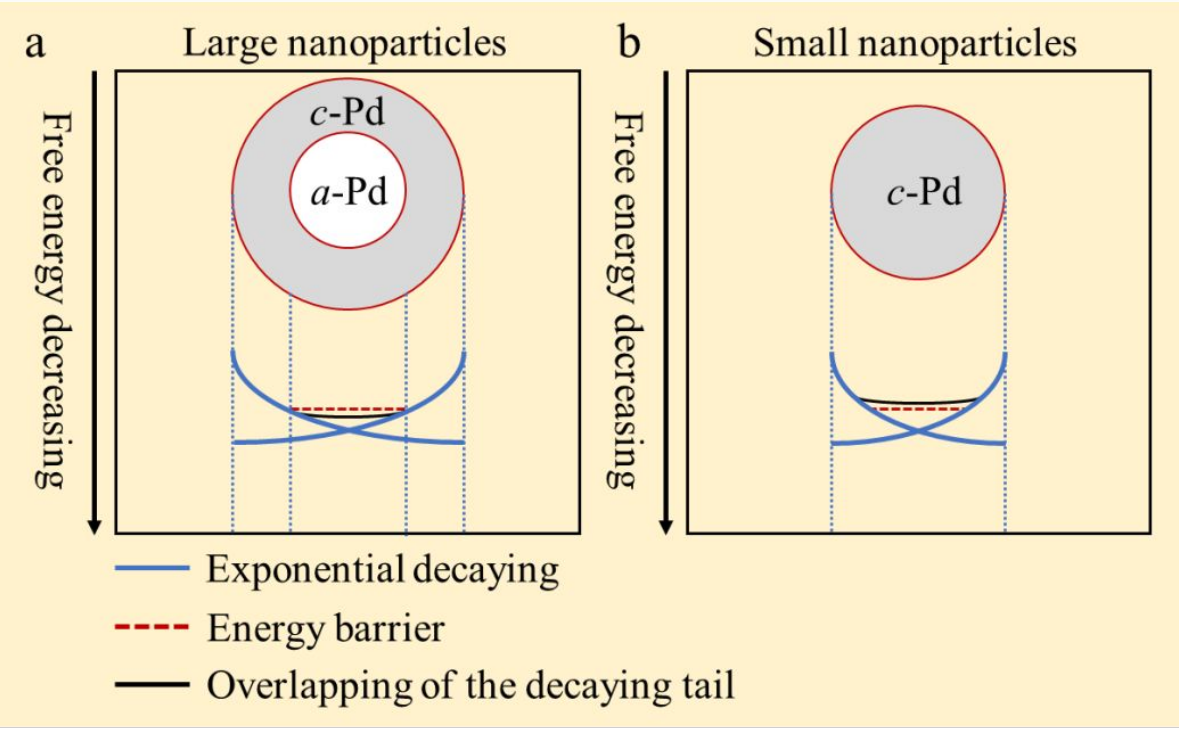

Figure S6. The tail overlapping of the symmetric decaying model for large nanoparticle $(R>7$ $\mathrm{nm}$, a) and small nanoparticle $(R<7 \mathrm{~nm}, \mathrm{~b})$, respectively. In our model, for larger nanoparticles, the overlapping of free energy (black solid line) in the interior of nanoparticles can not surpass the energy barrier for amorphous to crystalline transition (red dotted line), thus the core maintains amorphous structure. Instead, smaller nanoparticles experience complete crystallization because of the higher overlapped free energy. 


\section{The caption of Movie S1.}

The in situ transformation process of $a$-Pd nanoparticles under the irradiation of electron beam.

The incubation stage is not included in this movie because there are no changes during this period.

The time elapse starts from the first frame in the movie. 